\title{
Inversión Extranjera Directa y Productividad en el Perú ${ }^{1}$
}

\author{
Foreign Direct Investment and Productivity in Peru
}

\begin{abstract}
Alexci Igor Chong Ríos ${ }^{2}$
RESUMEN

El objetivo del presente estudio es encontrar evidencia empírica si la IED medida a través de empresas que tienen $15 \%$ a más de capital extranjero ha contribuido a mejorar la productividad de la industria en general. Así como verificar si estas empresas tienen mayor rentabilidad que las empresas con capitales nacionales. Por otro lado, se busca encontrar evidencia si existen spillovers de productividad hacia las empresas nacionales. El estudio encuentra: empresas con capital extranjero con mayor productividad y presencia de spillovers desde empresas con capital extranjero mayor a 50\%. Menor a este porcentaje, no existe significancia estadística.

Palabras clave: Inversión extranjera directa (IED); productividad laboral; spillovers; Bolsa de Valores de Lima (BVL); empresas multinacionales (EMN); modelo clásico de regresión lineal (MCRL).
\end{abstract}

JEL: F61

\footnotetext{
1 El estudio busca encontrar evidencia si la presencia de la inversión extranjera directa ha contribuido a mejorar mediante "spillovers" la productividad de las empresas locales y si estas tiene mayor productividad que las empresas extranjeras. El presente artículo es de investigación científica.

2 Economista, Ingeniero en Industrias Alimentarias y Magister en Finanzas por la Universidad Nacional de la Amazonía Peruana. Egresado del Doctorado en Gestión Económica Global por la Universidad Nacional Mayor de San Marcos. Docente universitario. (e-mail: alexci.chong@unmsm.edu.pe).

(C) Los autores. Este artículo es publicado por Pensamiento Crítico de la Facultad de Ciencias Económicas, Universidad Nacional Mayor de San Marcos. Este es un artículo de acceso abierto, distribuido bajo los términos de la licencia Creative Commons Atribucion - No Comercia_Compartir Igual 4.0 Internacional. (http://creativecommons.org/licenses/by-nc-sa/4.0/) que permite el uso no comercial, distribución y reproducción en cualquier medio, siempre que la obra original sea debidamente citada.
} 


\section{ABSTRAC}

The objective of this study is to find empirical evidence if the FDI measured through companies that have $15 \%$ more than foreign capital has contributed to improve the productivity of the industry in general. As well, as verify if these companies have greater profitability than companies with national capitals do. On the other hand, we seek to find evidence if there are spillovers of productivity towards national companies. The study finds companies with foreign capital with higher productivity and presence of spillovers from companies with foreign capital greater than $50 \%$. Less than this percentage, there is no statistical significance.

Palabras clave: Inversión extranjera directa (IED); productividad laboral; spillovers; Bolsa de Valores de Lima (BVL); empresas multinacionales (EMN); modelo clásico de regresión lineal (MCRL).

JEL: F61.

\section{Introducción}

Ciertamente las aportaciones teóricas sobre los beneficios de la presencia de la IED son extensas y favorables. Sin embargo, existen varios estudios empíricos que demuestran lo contrario, por lo que el debate aún continúa en agenda. Los estudios seminales para América Latina se llevaron a cabo en México. Reyes, Romo y Bazdresch(2004) condensan los estudios en dos oleadas. La primera,que replica los estudios de Caves (1974) y Globerman (1979),son realizadas porBlomström y Persson (1983), Blomström y Wolff (1994), Kokko (1994) quienes mediante técnicas de corte transversal evidencian existencia de una relación positiva y estadísticamente significativa entre la productividad del trabajo de las plantas locales y la presencia extranjera con conclusiones de existencia de derramas de eficiencia. La característica principal de este tipo de estudios es que utiliza la productividad laboral de las empresas domésticas como variable dependiente en las estimaciones econométricas y como una medida de eficiencia técnica. Se trataba de observar la forma en que cambia la productividad de la mano de obra en los establecimientos de propiedad doméstica ante la presencia de empresas subsidiarias de firmas multinacionales.

Los estudios de la segunda oleada son muy diferentes a los de la primera en varios aspectos. Primero, difieren en cuanto al diseño de la investigación. En segundo lugar, difieren en cuanto al método economé- 
trico empleado. En tercer término, son distintos de acuerdo al nivel de desagregación de los datos utilizados y periodo abarcado en el estudio. Un cuarto aspecto que hace diferentes los dos grupos de estudios son los resultados y, finalmente, contrastan al mostrar evidencia sobre la realización de otros tipos de derramas y al enfatizar en los mecanismos de transmisión que diferían de las anteriores por su ampliación a técnicas de datos de panel. Dentro de este segundo grupo de estudios se encuentran los siguientes: Aitken, Harrison y Lipsey (1996); Aitken, Hanson y Harrison (1997); Grether (1999); Romo (2002); y Domínguez y Brown (2004). Contrariamente a los estudios de la primera oleada, los estudios de la segunda oleada concluyen que existen derramas de productividad estadísticamente insignificantes o nulas como en Grether (1999), Romo (2004) y Domínguez y Brown (2004). Finalmente, Gorg y Greenaway (2004) mediante una revisión contextual reportan lo siguiente: de 40 estudios de spillovers de productividad en industrias de manufacturas para países desarrollados, en desarrollo y economías en transición, 19 reportan significancia estadística y efectos positivos de spillovers horizontal (8 de ellos utilizan datos de panel y 11 de corte transversal), 15 estudios no encuentran significancia estadística y 6 encuentran evidencias negativas de productividad en economías en transición.

Estas diferencias en los resultados también se ven afectadas con incertidumbre en términos de recomendación de políticas públicas. Las exoneraciones fiscales y aduaneras que se otorgan a multinacionales extranjeras es considerado innecesario por cuanto son empresas que se instalan con avances tecnológicos y financieros en orden superior a las empresas nacionales, más aún cuando repatrian gran parte de sus utilidades a sus matrices extranjeras y experimentan acelerados retornos de inversión. Por otra parte, es considerado por varios investigadores como vehículos de importantes flujos de divisas que aportan a la creación de empleos, incremento de recaudación tributaria, aumento de la formación bruta de capital y representan el efecto crowding in del esfuerzo del estado mediante el gasto público a fin de propiciar la atracción de las mismas. Más aun, considerando los efectos spillovers (contagios, derrames o desbordamientos) que propicia la inversión extranjera directa en aspectos como transferencia tecnológica, nuevos procesos, asistencia técnica, competencia, capacitación de capital humano y formación gerencial en beneficio de las empresas nacionales. 
Debido a la nula medición en el Perú sobre los impactos de las multinacionales sobre las firmas nacionales, el objetivo del presente artículo es aportar evidencia empírica en la productividad de las empresas y presencia de desbordamientos de productividad. Para tal fin, surgen dos interrogantes específicas: Primero: ¿las empresas extranjeras tienen mayor productividad laboral que las empresas nacionales? Segundo: ¿La presencia de empresas extranjeras a través de spillovers tiene efectos positivos sobre la productividad de las empresas nacionales? El objetivo del presente estudio es dar una respuesta con contenido empírico a estas interrogantes.

El estudio está relacionado a investigaciones previas como el de Blomström y Persson (1983), Blomström y Wolff (1994) y Kokko (1994) y en particular a un artículo de Fujii (2004) sobre la IED y la productividad en México.

El estudio se divide en las siguientes secciones: La sección 2 presenta la metodología aplicada, la sección 3 presenta el marco teórico, la sección 4 analiza los resultados y discusión, la sección 5 presenta las conclusiones y, finalmente la sección 6 presenta las recomendaciones.

\section{Metodología}

El estudio sigue un enfoque eminentemente cuantitativo del tipo correlacional y explicativo. El estudio se enmarca dentro del diseño no experimental, por cuanto no manipula variables. Es del tipo transversal ya que los datos son recolectados en un momento específico en el tiempo, años 2016 y 2017.

La muestra está conformada por 86 empresas representativas correspondiente a los sectores extractivo, de transformación y de servicios cuyos resultados de gestión económica y financiera se encuentran publicados en el portal WEB de la bolsa de valores de Lima (BVL). Al 31 de diciembre del 2017, existen 273 empresas que listan en la BVL. Para el estudio se excluyeron empresas que realizan inversiones en cartera, empresas en liquidación y/o reestructuración, fondos de inversión, seguros, administradoras de fondos de pensiones y otras que no actualizaron información. La muestra el total de empresas sujetas a medición. 
Pensamiento Crítico Vol. 24. $\mathrm{N}^{\circ} 1$

Tabla 1

PERU: Empresas seleccionadas que listan en la BVL

\begin{tabular}{|c|c|c|c|c|c|}
\hline $\mathbf{N}^{\circ}$ & Empresa & $\mathbf{N}^{\circ}$ & Empresa & $\mathbf{N}^{\circ}$ & Empresa \\
\hline 1 & Azucarera Chucarapi SA & 30 & Compañía Good Year del Perú SA & 59 & Luz del Sur SA \\
\hline 2 & $\begin{array}{l}\text { Agricola y Ganadera Chavin de } \\
\text { Huantar }\end{array}$ & 31 & Creditex SAA & 60 & Manufacturas Record SA \\
\hline 3 & Agroindustrial Paramonga SAA & 32 & Cerro Verde SA & 61 & Metalurgica Peruana SA Mepsa \\
\hline 4 & Agroindustrial Laredo SAA & 33 & Eco Acuicola SAC y subsidiaria & 62 & Michel y Cía SA \\
\hline 5 & Agroindustrial AIB SA & 34 & Electro Sur Este SAA & 63 & Minsur SA \\
\hline 6 & Agroindustrias San Jacinto SA & 35 & Electro Puno SAA & 64 & Mineras Atacocha SAA \\
\hline 7 & Agroindustria Cayalti SA & 36 & Empresa Siderurgica del Perú & 65 & Motores diessel andinos SAA \\
\hline 8 & Alicorp SAA & 37 & Hidrandina SA & 66 & Nessus Hoteles Perú SA \\
\hline 9 & Austral Group SAA & 38 & Empresa Agricola San Juan SA & 67 & $\begin{array}{l}\text { Peruana de moldeados } \\
\text { Pamolsa }\end{array}$ \\
\hline 10 & Agrokasa Holdings & 39 & Empresa Agricola Sintuco SA & 68 & Pesquera Exalmar SAA \\
\hline 11 & BPO Consulting SAC & 40 & Empresa azucarera El Ingenio SA & 69 & Pesquera Hayduk SA \\
\hline 12 & Backus SA & 41 & Enel Distribución Perú SAA & 70 & Perubar SA \\
\hline 13 & Bayer SA & 42 & Exsa SA & 71 & Petróleos del Perú SA \\
\hline 14 & Cia de Minas Buenaventura SAA & 43 & Ferreycorp & 72 & Quimpac SA \\
\hline 15 & Cartavio SAA & 44 & Fabrica Acumuladores Etna SA & 73 & $\begin{array}{l}\text { Representación Química } \\
\text { Europea SAC }\end{array}$ \\
\hline 16 & Camposol & 45 & Fabrica Peruana Ethernit SA & 74 & Refinería la Pampilla SAA \\
\hline 17 & Casagrande SAA & 46 & Filamentos Industriales & 75 & Saga Falabella SA \\
\hline 18 & Cementos Pacasmayo SAA & 47 & Hermes Transportes Blindado & 76 & Servicios Generales Saturno SA \\
\hline 19 & Cervezeria San Juan SA & 48 & Hidrostal SA & 77 & $\begin{array}{l}\text { Shougang Generación Eléctrica } \\
\text { SAA }\end{array}$ \\
\hline 20 & Compañía Minera Santa Luisa SA & 49 & Indeco SA & 78 & Shougang Hierro Perú SAA \\
\hline 21 & Compañía Universal Textil SA & 50 & Industrias del Envase SA & 79 & $\begin{array}{l}\text { Sociedad electrica del Sur } \\
\text { Oeste SA }\end{array}$ \\
\hline 22 & $\begin{array}{l}\text { Consorcio Industrial de } \\
\text { Arequipa SA }\end{array}$ & 51 & $\begin{array}{l}\text { Industria Electro QuÍmica } \\
\text { IEQSA SA }\end{array}$ & 80 & Sociedad Minera Corona SA \\
\hline 23 & $\begin{array}{l}\text { Corporación de Aceros } \\
\text { Arequipa SA }\end{array}$ & 52 & Intradevco Industrial SA & 81 & Sociedad Minera El Broncal SAA \\
\hline 24 & Corporación de Cerámica SA & 53 & Invertur SA & 82 & $\begin{array}{l}\text { Southerm Perú Copper } \\
\text { Corporation }\end{array}$ \\
\hline 25 & $\begin{array}{l}\text { Corporación Lindley y } \\
\text { subsidiarias }\end{array}$ & 54 & Intursa & 83 & Tecnología de Alimentos TASA \\
\hline 26 & Cosapi SA & 55 & Jockey Plaza Shopping Center SAA & 84 & Unión andina de cementos SA \\
\hline 27 & Compañía Minera Poderosa SA & 56 & Laive SA & 85 & Volcán SA \\
\hline 28 & $\begin{array}{l}\text { Cía Mina San Ignacio de } \\
\text { Morococha SAA }\end{array}$ & 57 & Leche Gloria SA & 86 & Yura y Subsidiarias SA \\
\hline 29 & Comercial del Acero SA & 58 & Lima Caucho SA & & \\
\hline
\end{tabular}

Fuente. Bolsa de Valores de Lima 


\section{Así mismo, la tabla 2 muestra las empresas que listan en bolsa clasifi- cadas por procedencia (nacional o extranjera) y por sectores (extractivas, de transformación y de servicios). El criterio para definir la procedencia está en función al capital accionario. Las informaciones de empresas son listadas de la página WEB de la BVL para los años 2016 y 2017).}

Tabla 2

PERÚ: Empresas seleccionadas por sector y procedencia

\begin{tabular}{|c|c|c|}
\hline $\mathbf{N}^{\circ}$ & Extractiva & \\
\hline 1 & Cía de Minas Buenaventura SAA & Extranjera \\
\hline 2 & Compañía Minera santa Luisa SAA & Extranjera \\
\hline 3 & Compañía Minera Poderosa SA & Extranjera \\
\hline 4 & Cía Mina San Ignacio de Morococha SA & Extranjera \\
\hline 5 & Cerro Verde SA & Extranjera \\
\hline 6 & Minsur SA & Nacional \\
\hline 7 & Mineras Atacicha SAA & Nacional \\
\hline 8 & Perubar SA & Extranjera \\
\hline 9 & Shougang Hierro Perú SAA & Extranjera \\
\hline 10 & Sociedad Minera Corona SA & Extranjera \\
\hline 11 & Sociedad Minera El Brocal SAA & Extranjera \\
\hline 12 & Southern Perú Copper Corporation & Extranjera \\
\hline 13 & Volcan SA & Extranjera \\
\hline $\mathbf{N}^{\circ}$ & Transformación & \\
\hline 1 & Azucarera Chucarapi SA & Nacional \\
\hline 2 & Agricola y Ganadera Chavin de Huantar & Nacional \\
\hline 3 & Agroindustrial Paramonga SAA & Nacional \\
\hline 4 & Agroindustrial Laredo SAA & Extranjera \\
\hline 5 & Agroindustrial AIB SA & Nacional \\
\hline 6 & Agroindustria San Jacinto SA & Nacional \\
\hline 7 & Agroindustria Cayalti SA & Nacional \\
\hline 8 & Alicorp SAA & Extranjera \\
\hline 9 & Austral Group SAA & Extranjera \\
\hline 10 & Agrokasa Holdings & Extranjera \\
\hline 11 & Backus SA & Extranjera \\
\hline 12 & Cartavio SAA & Nacional \\
\hline 13 & Camposol & Extranjera \\
\hline 14 & Casagrande SAA & Nacional \\
\hline 15 & Cementos Pacasmayo SAA & Extranjera \\
\hline 16 & Cervezeria San Juan SA & Nacional \\
\hline 17 & Compañía Universal Textil SA & Nacional \\
\hline 18 & Compañía Universal de Arequipa SA & Nacional \\
\hline 19 & Corporación Aceros Arequipa SA & Nacional \\
\hline 20 & Corporación Cerámica SA & Nacional \\
\hline 21 & Corporación Lindley y subsidiarias & Extranjera \\
\hline 22 & Compañía Good Year del Perú SA & Extranjera \\
\hline 23 & Creditex SAA & Extranjera \\
\hline 24 & Eco Acuicola SAC y subsidiaria & Nacional \\
\hline 25 & Empresa Siderurgica del Perú & Extranjera \\
\hline
\end{tabular}


Pensamiento Crítico Vol. 24. № 1

\begin{tabular}{|c|c|c|}
\hline 26 & Empresa Agricola San Juan SA & Nacional \\
\hline 27 & Empresa Agricola Sintuco SA & Nacional \\
\hline 28 & Empresa Azucarera El Ingenio SA & Nacional \\
\hline 29 & Exsa SA & Nacional \\
\hline 30 & Fabrica Acumuladores Etna SA & Nacional \\
\hline 31 & Fabrica Peruana Ethernit SA & Nacional \\
\hline 32 & Filamentos Industriales & Nacional \\
\hline 33 & Hidrostal SA & Extranjera \\
\hline 34 & Indeco SA & Extranjera \\
\hline 35 & Industrias del Envase SA & Extranjera \\
\hline 36 & Industria Electro Quimica IEQSA SA & Extranjera \\
\hline 37 & Intradevco Industrial SA & Extranjera \\
\hline 38 & Laive SA & Extranjera \\
\hline 39 & Leche Gloria SA & Nacional \\
\hline 40 & Lima Caucho SA & Extranjera \\
\hline 41 & Manufacturas Record SA & Extranjera \\
\hline 42 & Metalurgica Peruana SA Mepsa & Extranjera \\
\hline 43 & Michel y Cia SA & Nacional \\
\hline 44 & Motores diessel andinos SA & Extranjera \\
\hline 45 & Peruana de moldeados Pamolsa & Extranjera \\
\hline 46 & Peruana Exalmar SAA & Extranjera \\
\hline 47 & Pesquera Hayduk SA & Extranjera \\
\hline 48 & Petroleos del Perú SA & Nacional \\
\hline 49 & Quimpac SA & Extranjera \\
\hline 50 & Representación Química Europea SAC & Extranjera \\
\hline 51 & Refinería la Pampilla S.A.A. & Extranjera \\
\hline 52 & Tecnólogica de Alimentos TASA & Nacional \\
\hline 53 & Union andina de cementos SA & Nacional \\
\hline 54 & Yura y subsidiarias SA & Nacional \\
\hline $\mathbf{N}^{\circ}$ & Servicios & \\
\hline 1 & BPO Consulting SAC & Nacional \\
\hline 2 & Bayer SA & Extranjera \\
\hline 3 & Cosapi SA & Nacional \\
\hline 4 & Comercial del Acero SA & Nacional \\
\hline 5 & Electro Sur Este SAA & Nacional \\
\hline 6 & Electro Puno SAA & Nacional \\
\hline 7 & Hidrandina SA & Nacional \\
\hline 8 & Enel Distribución Perú SAA & Extranjera \\
\hline 9 & Ferreycorp & Nacional \\
\hline 10 & Hermes Transportes Blindado & Nacional \\
\hline 11 & Inventur SA & Extranjera \\
\hline 12 & Intursa & Nacional \\
\hline 13 & Jockey Plaza Shopping Center SAA & Extranjera \\
\hline 14 & Luz del Sur SA & Extranjera \\
\hline 15 & Nessus Hoteles Perú SA & Extranjera \\
\hline 16 & Saga Fallabela SA & Extranjera \\
\hline 17 & Servicios Generales Saturno SA & Extranjera \\
\hline 18 & Shougang Generación Eléctrica SAA & Extranjera \\
\hline 19 & Sociedad electrica del Sur Oeste SA & Nacional \\
\hline
\end{tabular}

Fuente. Bolsa de Valores de Lima. 
La tabla 3 muestra el resumen por empresas extrajeras local y nacional, así como por el sector al que pertenece.

El modelo desarrollado es el modelo de regresión múltiple ANCOVA que utiliza variables continuas y categóricas. Se define la variable dependiente en términos de la productividad laboral del total de empresas seleccionadas (PROD). Esta variable está representada como el cociente de las ventas totales de la empresa y la cantidad de trabajadores durante el periodo anual para cada empresa. Para poder operacionalizar introducimos una variable dummy (INV) el cual toma el valor 1 para empresas con presencia de capital accionario extranjero mayor o igual al 15\% y el valor 0 para empresas nacionales. El porcentaje fijado del $15 \%$ permite a un accionista tener presencia en el directorio y como consecuencia, decisiones en la gestión empresarial. De acuerdo a la teoría un coeficiente positivo de la variable dummy indica que las empresas con capital accionario extranjero tienen mayor productividad.

Las variables explicativas son la presencia de empresas con capitales nacionales y extranjeros (INV), la calidad de trabajadores por empresa (SKILL)y la concentración de mercado (HERF).

Para la construcción de la variable SKILL (del inglés destreza) clasificamos dos tipos de trabajadores: Para las empresas extractivas y de transformación, cuello blanco (funcionarios y empleados) y cuello azul (obreros). Mientras que, para las empresas de servicios, cuello blanco (funcionarios y ejecutivos) y cuello azul (empleados). La variable es definida como el cociente de trabajadores de cuello blanco y cuello azul. En la medida que el personal sea de cuello azul, indica personal menos capacitado, por tanto, la productividad laboral será menor. El coeficiente de la variable SKILL se espera que sea positiva, la cual se traduce en que un personal mayormente calificado, incrementa la productividad.

Tabla 3

PERÚ: Resumen de empresas

\begin{tabular}{ccccc} 
Procedencia & Extractivas & Transformación & Servicios & Total \\
Locales & 2 & 27 & 9 & 38 \\
Extranjeras & 11 & 27 & 10 & 48 \\
Total & 13 & 54 & 19 & 86 \\
\hline
\end{tabular}

Fuente. Elaboración propia 
La construcción de la variable concentración de mercado a través del índice Herfindahl-Hirshman(HERF) busca encontrar el efecto de la competencia en la industria en general. El objetivo es medir el efecto de la concentración de trabajadores en la industria. Una concentración elevada se entiende menor capacidad competitiva entre las distintas empresas de medición. Para el cálculo se divide el total de trabajadores por empresa entre el total de trabajadores de las 86 empresas seleccionadas. Un coeficiente positivo indica una igualdad de concentración laboral y consecuentemente aumento de la productividad laboral. Este índice es definido como la concentración de la cuota mercado del sector para todas las empresas que lo componen. Así, valores mínimos del ÍndiceHerfindahlindicanigualdad de concentración de mercado, y un valor máximo estará ligado a una concentración elevada.

El modelo empírico desarrollado para determinar la existencia de mayor productividad por parte de las empresas está representado mediante la siguiente ecuación.

$$
\operatorname{PROD}_{t}=\beta_{0}+\beta_{1} I N V_{1 t}+\beta_{2} \mathrm{SKILL}_{2 t}+\beta_{3} \mathrm{HERF}_{3 t}+\mu
$$

La tabla 4 muestra la descripción de las variables.

Tabla 4

Descripción de variables en la estimación

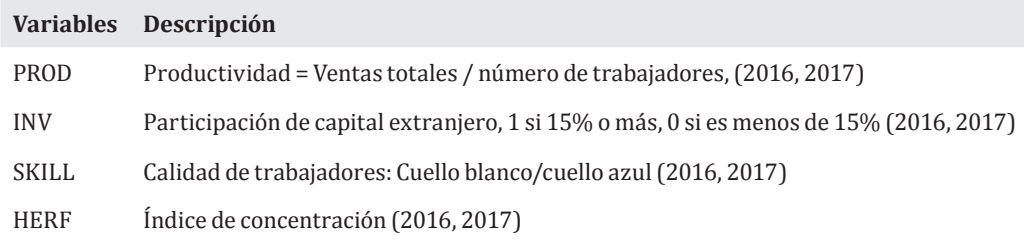

Elaboración propia

A efectos de evaluar los spillovers de productividad laboral realizamos los siguientes cambios al modelo original.

1. Utilizamos como variable dependiente la productividad laboral solo para las empresas nacionales, sin considerar las empresas extranjeras. La variable la definimos como $P R O D_{L^{*}}$ 
2. Construimos una nueva variable denominada VTASEXT que representa el cociente entre el porcentaje de ventas de empresas extranjeras dividido entre el total de ventas de la industria en general.

3. Construimos una nueva variable denominada TRAEXT que representa el cociente entre el porcentaje de trabajadores en empresas extranjeras y el total de trabajadores de la industria en general.

4. Para verificar la consistencia de los resultados se incluyen dos variables adicionalesROBVEXT y ROBTEXT, porcentaje de ventas y trabajadores de empresas con acciones mayoritariamente extranjero (al menos 50\% de IED)

El signo positivo de la variable que representa la participación de las empresas extranjeras en las ventas totales de la industria (VTASEXTR) se interpretará como indicador de la existencia de un efecto positivo de la presencia de las empresas extranjeras en las diferencias en la productividad de las empresas nacionales. En otros términos, de acuerdo con la teoría, si VTASEXT o TRAEXTtienen coeficientes positivos y significativos, existen spillovers de productividad. El modelo empírico es el siguiente:

$$
\begin{gathered}
\text { PROD }_{\mathrm{L}}=\beta_{0}+\beta_{1} \operatorname{VTASEXT~}_{1 \mathrm{~T}}+\beta_{2} \text { TRAEXT }_{2 \mathrm{~T}}+\beta_{3} \text { ROBVEXT }_{3 \mathrm{~T}}+ \\
\beta_{4} \operatorname{ROBTEXT}_{4 \mathrm{t}}+\mu(2)
\end{gathered}
$$

El detalle de las variables se resume en la tabla 5.

Tabla 5

Variables definidas en la estimación de spillovers

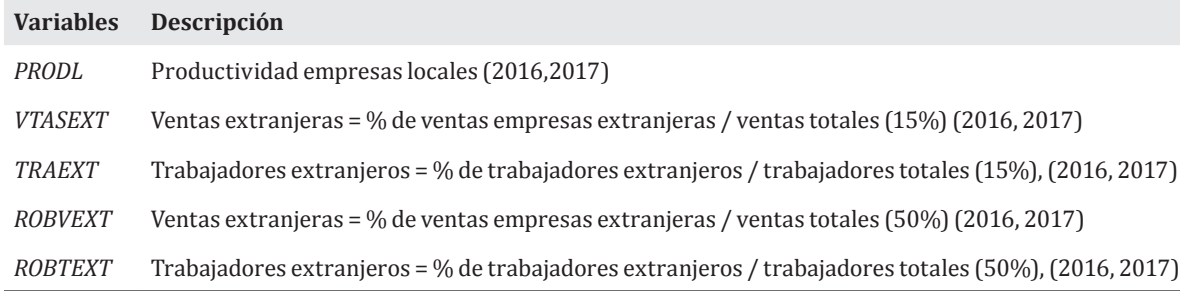

Elaboración: Propia

La información obtenida mediante una revisión detallada de las86 empresas representativas en el Perú de diversos sectores que listan en 
la BVL es procesada en el programa estadístico Stata 14 y Eviews 8. La muestra de empresas utilizadas en el estudio es de distintos tamaños y consecuentemente heterogénea. Cuando se incluyen unidades heterogéneas en un análisis estadístico de corte transversal debe tenerse presente el efecto del tamaño o escala que pueden generar problemas de heterogeneidad (asimetría y heteroscedasticidad). A fin de corregir el problema se adopta el modelo log-lineal que permite sacar logaritmo a la variable regresada.

$$
\operatorname{Ln}\left(\operatorname{PROD}_{\mathrm{t}}\right)=\beta_{0}+\beta_{1} \mathrm{INV}_{1 \mathrm{t}}+\beta_{2} \mathrm{SKILL}_{2 \mathrm{t}}+\beta_{3} \mathrm{HERF}_{3 \mathrm{t}}+\mu(3)
$$

La ausencia de tratar los valores observados de las empresas, puede potencialmente producir problemas de heteroscedasticidad, un problema común en datos de corte transversal ya que los intervalos de confianza que permiten una correcta inferencia estadística serán muy amplios. Por tanto, las pruebas t y $\mathrm{F}$ pueden reportar resultados imprecisos con coeficientes estadísticamente no significativos y que de hecho pueden resultar significativos si se establece intervalos de confianza correctos.

Para evaluar la consistencia y estructura de los modelos conviene efectuar un conjunto de pruebas de validación de los resultados obtenidos. Con este propósito las pruebas de diagnóstico se establecen a los siguientes test: Normalidad, heteroscedasticidad y prueba de forma funcional de Ramsey. El test de normalidad se analiza mediante el indicador Jarque Bera el cual sostiene la hipótesis nula que la regresada estimada en el modelo adopta distribución normal. La prueba de heteroscedasticidad, aplica el test de White el cual debe evidenciar que sí el chi- cuadrado obtenido es menor al chi-cuadrado critico a un nivel de $95 \%$ de confianza, se acepta la hipótesis nula de homoscedasticidad. El test de Ramsey presenta la hipótesis nula de que no existen variables omitidas en el modelo siempre que el valor $\mathrm{F}$ estimado es menor al $\mathrm{F}$ tabulado de acuerdo a los grados de libertad.

\section{Marco Teórico}

\subsection{Modalidades de IED}

Ronderos (2010) indica que las empresas multinacionales invierten en un país a través de dos modalidades: mediante greenfield investment y fu- 
siones y adquisiciones. La primera consiste en que una empresa matriz construye "desde cero" las instalaciones para una industria a través de levantamiento de nuevas instalaciones, sea un centro de fabricación y/o transformación, un departamento de logística y distribución, nueva unidad de prestación de servicios a fin de operar y en el país de su interés. La segunda se realiza mediante compras de acciones o alianzas de propiedad mixta sobre empresas ya existentes. En el Perú este ha sido la forma más predominante durante la década de los 90 mediante la transferencia de empresas estatales a través de la privatización.

La IED puede ser horizontal o vertical. Cuando una empresa toma la actividad de una misma etapa de la cadena de valor de su propio país y la duplica en un país huésped por medio de IED, llamamos a esto IED horizontal. Si una empresa se mueve hacia arriba o hacia abajo en diferentes etapas de la cadena de valor en un país huésped por medio de la IED, calificamos a esto como IED vertical.

Sasidharan y Ramanathan (2007) citado por Jiménez y Rendón (2011) señalan que las multinacionales transfieren tecnología a través de mecanismos como: licencias, comercios, subcontrataciones, franquicias o alianzas estratégicas. Los efectos indirectos que conlleva este tipo de inversiones en la productividad de las empresas nacionales se conocen como spillovers. Cuando la entrada de las empresas extranjeras genera un incremento de productividad en las empresas locales que pertenecen a la misma industria se denominan spillovers horizontales o intraindustrial. En cambio, si el incremento de productividad se da a nivel de la relación entre los proveedores y las empresas extranjeras ésta se conoce como spillovers verticales o interindustrial.

\subsection{Evidencia empírica}

Ortiz y otros (2019), en un artículo sobre el efecto de la IED y libertad económica como determinantes del crecimiento económico en Ecuador para los años 1980 -2017 encuentran relación positiva en dos direcciones: IED - PBI e IED y formación bruta de capital. Guerra-Borge(2001), sostiene inexistencia de evidencia empírica que permita comprobar relación entre IED y el crecimiento económico. Cita el caso de Corea del Sur quien experimentó un extraordinario crecimiento con cargo al ahorro interno y en menor medida a capital foráneo. Suanes y Roca (2015) afirman encontrar 
evidencia favorable entre IED y crecimiento económico pero desfavorable en cuanto a desigualdad de ingresos en la población. Para Maminingi y Karem (2018), la literatura económica no muestra unanimidad sobre el impacto del crecimiento y presencia de IED. Señalan que estudios realizados por Schneider y Frey (1985) mostraron relaciones positivas y con significancia estadística entre la IED y crecimiento, pero en las investigaciones realizadas por Nigh (1986) y Balasubramanyam, Salisu y Sapsford (1996) concluyeron que no existenefectos significativos de IED sobre el crecimiento económico. Villena (2013) realizó un estudio de consultoría para el Gobierno de Chile con el objetivo de determinar y medir el aporte real que existió desde 1980 al 2011 acerca de la Inversión Extranjera Directa sobre diversos aspectos de la economía chilena. Los resultados encontrados demuestran que la IED ha ejercido un efecto positivo en el crecimiento económico.

A nivel empresas, Blomström (1989) señala que frecuentemente se afirma que los países anfitriones ganan beneficios tecnológicos a través de spillovers ${ }^{3}$ de productividad mediante la inversión extranjera directa. Tales spillovers se traslapan de diferentes formas. Las corporaciones multinacionales pueden, por ejemplo, incrementar la competitividad que se traduce en métodos más eficientes en la cadena de valor. A la vez, estimula a las empresas locales a adoptar más rápidamente tecnologías específicas y mejorar el performance de trabajadores y directivos. Considerando el hecho que los componentes de los spillovers son diversos y difíciles de medir existen algunos métodos empíricos que miden tales efectos.

Blomström y Persson (1983) sobre la industria manufacturera mexicana, realizan un análisis a nivel sectorial que sugiere un impacto positivo de la presencia de capital externo sobre la productividad de la industria, así como un impacto positivo de la presencia de empresas extranjeras sobre la productividad de las domésticas. Es decir, en ambos casos, se comprueba la presencia de spillovers de productividad.

Fujii (2004), en un estudio sobre el impacto de la IED en México, utiliza 174 empresas del sector manufacturero y encuentra que las empresas con capitales extranjeros tienen un impacto favorable sobre la productividad, pero no tiende a incrementar la productividad de las empresas domésticas.

3 Refiere a efectos de desbordamientos, arrastre o contagio. 
Jiménez y Rendón (2011) estudiaron la presencia de spillovers de productividades horizontales y verticales para la industria manufacturera colombiana para el periodo comprendido entre 2001-2005. Mediante un modelo de datos de panel la estimación del modelo muestra spillovers horizontales negativos y verticales positivos.

\subsection{Comportamiento de la IED en Perú}

Es escasa la información sobre estudios empíricos en el Perú. Los estudios realizados son generalmente de carácter descriptivo. Araoz, Casas y Noriega (2002), en un estudio sobre la atracción de la IED en el Perú destacan la importancia de la IED a través de los encadenamientos productivos, generadores de industrias conexas y clusters empresariales, la adquisición de nuevas tecnologías y el aumento en el nivel de empleo y de divisas.

En el Perú es de conocimiento público y muy difundido que la presencia de la inversión extranjera directa tiene la virtud y potencia de generar empleo, aumentar la productividad a través de la tecnología, innovación y conocimiento especializado y ser motor del crecimiento económico. En aras a conseguir presencia de capitales extranjeros, nuestro país tiene a la fecha 21 tratados de libre comercio y acuerdos comerciales y ha promocionado la seguridad jurídica y el respeto a la inversión privada.

Sin embargo, el balance neto de entradas y salidas de capitales ha sido desfavorable. La tabla 6 muestra un déficit desde 1995 al 2017 en U.S. \$ 6,989 millones de dólares entre los ingresos por IED y los egresos privados consignados en la renta de factores y cuenta financiera del sector privado de la balanza de pagos. La mayor participación en las cifras de egresos privados consignados en la tabla está representada por la repatriación de utilidades de las empresas filiales en el Perú al país de origen.

Esta magnitud de repatriación de utilidades es aún mayor en U.S. \$ 18,455 millones de dólares durante el periodo 2005 al 2017 con tendencia a la salida de divisas. Esto tendría una explicación en que las empresas extranjeras ya alcanzaron un nivel de madurez en la economía receptora. La figura 1 muestra la evolución de los flujos de IED y egresos privados. 
Tabla 6

PERÚ: Flujo de IED y egresos privados, 1995 - 2017. (Millones de dólares)

\begin{tabular}{|c|c|c|c|c|c|}
\hline Años & Inv. Drecta Extranjera & Egresos Privados & Años & Inv. Drecta Extranjera & Egresos Privados \\
\hline 1995 & $2,557.0$ & 833.0 & 2008 & $6,923.7$ & $8,346.0$ \\
\hline 1996 & $3,471.1$ & 676.0 & 2009 & $6,430.7$ & $8,172.0$ \\
\hline 1997 & $2,139.3$ & 926.0 & 2010 & $8,454.6$ & $10,715.0$ \\
\hline 1998 & $1,643.9$ & 221.0 & 2011 & $7,665.0$ & $12,323.0$ \\
\hline 1999 & $1,940.0$ & 41.0 & 2012 & $11,918.0$ & $11,591.0$ \\
\hline 2000 & 809.7 & 345.0 & 2013 & $9,298.0$ & $9,889.0$ \\
\hline 2001 & $1,144.3$ & 131.0 & 2014 & $7,885.0$ & $8,497.0$ \\
\hline 2002 & $2,155.8$ & 478.0 & 2015 & $8,272.0$ & $5,812.0$ \\
\hline 2003 & $1,335.0$ & $1,112.0$ & 2016 & $6,863.0$ & $7,268.0$ \\
\hline 2004 & $1,599.0$ & $2,566.0$ & 2017 & $6,769.0$ & $9,297.0$ \\
\hline 2005 & $2,578.7$ & $4,030.0$ & & & \\
\hline 2006 & $3,466.5$ & $6,742.0$ & & & \\
\hline 2007 & $5,491.0$ & 7,788.0 & & & \\
\hline
\end{tabular}

Fuente: BCR, Proinversión

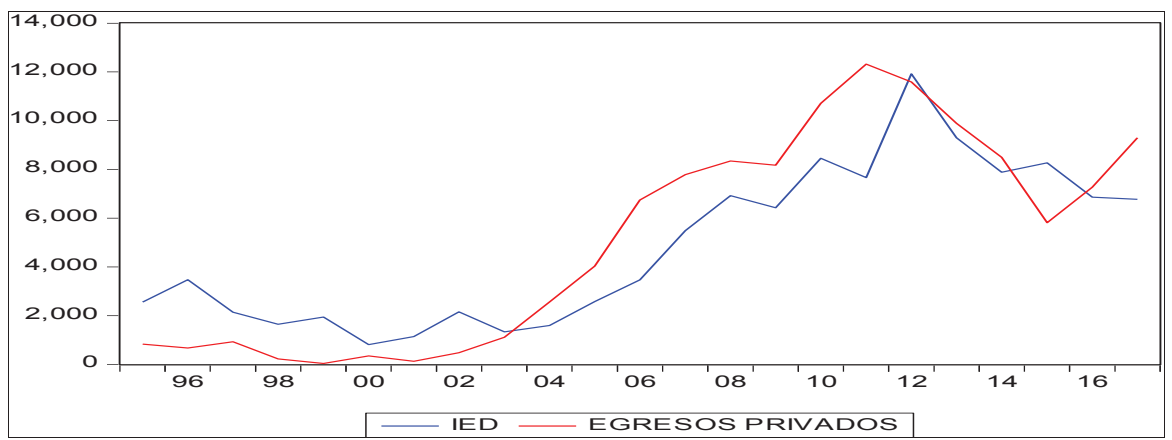

Figura 1. PERÚ: Flujo de IED y egresos privados, 1995 -2017. (Millones de dólares)

Fuente. Elaboración propia

Huerta (2010) determinó la relación existente entre la localización de la IED en el Perú y factores como el tamaño de mercado, costos laborales, inversión pública en infraestructura y la incertidumbre económica por paralizaciones. Utilizando el enfoque ecléctico de Dunning y el modelo doble log, encontró que el principal factor que explica la decisión de localización de la IED es el tamaño de mercado representado por la demanda 
interna sin considerar la inversión fija pública. Un segundo determinante es el costo laboral representado por el sueldo real de Lima metropolitana. Factores como inversión pública, gastos de capital e incertidumbre económica muestran significancia estadística y son determinantes, en menor proporción, en la localización.

\section{Resultados y Discusión}

La tabla 7 muestra los estadísticos descriptivos de la muestra. Las empresas comprendidas en el sector transformación registran valores mínimos y máximos extremos en relación a los otros sectores. Como consecuencia una mayor variación. El sector servicios observa mayor productividad laboral media para ambos años.

Tabla 7

Características de la muestra

\begin{tabular}{lcccccccc} 
& \multicolumn{2}{c}{ Valor Mínimo } & \multicolumn{2}{c}{ Valor Máximo } & \multicolumn{2}{c}{ Media } & \multicolumn{2}{c}{ Desviación Estándar } \\
& $\mathbf{2 0 1 6}$ & $\mathbf{2 0 1 7}$ & $\mathbf{2 0 1 6}$ & $\mathbf{2 0 1 7}$ & $\mathbf{2 0 1 6}$ & $\mathbf{2 0 1 7}$ & $\mathbf{2 0 1 6}$ & $\mathbf{2 0 1 7}$ \\
Extractivas & 144.96 & 128.76 & $1,803.28$ & $2,268.17$ & 699.33 & 832.7 & 516.96 & 647.79 \\
Transformación & 9.28 & 2.98 & $12,825.51$ & $18,163.23$ & 887.92 & $1,035.32$ & $1,848.36$ & $2,548.41$ \\
Servicios & 21.94 & 13.79 & $4,678.59$ & $4,687.48$ & $1,274.29$ & $1,262.43$ & $1,445.51$ & $1,436.86$ \\
Universo de Empresas & 9.28 & 2.98 & $12,825.51$ & $18,163.23$ & 944.77 & $1,054.87$ & $1,626.65$ & $2,136.19$ \\
\hline
\end{tabular}

Elaboración: Propia

La tabla 8 muestra los resultados de la estimación para determinar el impacto que tiene la presencia de empresas con capitales accionarios extranjeros sobre la productividad de la industria en general y de las empresas nacionales en particular

Los resultados del modelo indican que en ambos periodos los coeficientes de las variables inversión extranjera y habilidad son positivos y significativos. Por tanto, impactan positivamente en la productividad laboral, mientras que el signo del coeficiente de la variable concentración de mercado es negativo y significativo, afectando la productividad de la industria. El signo positivo del coeficiente de la variable $I N V$ indica que las empresas con presencia de capital extranjero tienden a ser más productiva que las empresas nacionales. La lectura numérica de la productividad laboral es explicada porcentualmente con respecto a la variable dummy $I N V$. Se genera el antilogaritmo del coeficiente de $I N V\left[0.605\left(\mathrm{e}^{0.605}-1\right)=\right.$ 
0.83], lo que indica que un incremento de una unidad en la productividad de las empresas extranjeras provoca un incremento del $83 \%$ en la productividad de la industria en general. Los resultados de la regresión están en el mismo plano con los estudios de la primera oleada de Blomström y Persson (1983) y Blomström (1986) que realizaron estudios con un diseño de corte transversal en la industria manufacturera mexicana correspondiente al año 1970 y 1978. En dichos estudios los autores encontraron una relación positiva y significativa entre la eficiencia de las empresas locales (medido como productividad laboral) y aquellas con participación extranjera. Fujii (2004), en una réplica de los estudios anteriores para el mismo país en el periodo 1993 - 1994 encontró que la presencia de capital extranjero tiene un coeficiente positivo y significativo, lo que implica que las empresas extranjeras tienden a ser más productivas, similares a estudios seminales.

Tabla 8

Resultados de la estimación

\begin{tabular}{|lrr|}
\hline & 2016 & 2017 \\
\hline INV & 0.605 & 0.613 \\
& ee $=0.273$ & $\mathrm{ee}=0.294$ \\
$(0.030)^{*}$ & $(0.041)^{*}$ \\
\hline SKILL & 0.243 & 0.259 \\
& $\mathrm{e}=0.078$ & $\mathrm{e}=0.089$ \\
& $(0.003)^{*}$ & $(0.004)^{*}$ \\
\hline HERF & -249.974 & -481.369 \\
& $\mathrm{ee}=97.17 \mathrm{ee}=137.15$ \\
$\mathrm{~g}$ & $(0.001)^{*}$ \\
\hline Constante & 5.522 & 5.583 \\
& $\mathrm{ee}=0.224$ & $\mathrm{ee}=0.242$ \\
Pruebas de diagnóstico & $(0.000)^{*}$ & $(0.000)^{*}$ \\
$\mathrm{~J} / \mathrm{B}$ & \multicolumn{2}{|c}{} \\
White & {$[0.95]$} & {$[8.59]$} \\
Ramsey & {$[0.68]$} & {$[0.72]$} \\
$\mathrm{R}^{2}$ & {$[0.03]$} & {$[0.08]$} \\
$\mathrm{F}$ & 0.2043 & 0.2385 \\
J & $(7.02)$ & $(8.56)$
\end{tabular}

$\mathrm{J} / \mathrm{B}=$ Prueba de Jarque Bera de Normalidad

White $=$ Prueba de white de heteroscedasticidad

Ramsey : Prueba de forma funcional

$\mathrm{F}=$ Prueba de significancia de las variables en conjunto

$*$ = Significancia al 95\% de confianza

Fuente. Elaboración propia

La variable SKILL denota signo positivo y significativo lo que corrobora de acuerdo a la literatura empírica que un mayor personal califi- 
cado repercute positivamente en la productividad laboral. Un aspecto importante a destacar, al margen de las características de este canal de transmisión de conocimiento, es que la transferencia de la tecnología de las empresas con capital extranjero a las empresas nacionales no sólo consiste en maquinaria, equipos o derechos de patentes, también se realiza a través de la formación de los empleados de las filiales extranjeras. Esta formación afecta a la mayoría de los niveles de empleados, desde los mandos operativos de bajo nivel hasta supervisores, o profesionales, con alto nivel cualitativo y administradores de nivel superior. Aunque estos últimos niveles desempleados son, inicialmente, cubiertos por expatriados, la participación de los empleados nacionales aumenta con el paso del tiempo.

Para el caso de la variable $H E R F$ el signo negativo indica que una mayor concentración de trabajadores repercute negativamente en la productividad laboral. En efecto, los datos muestran que para el año 2016 seis empresas concentran el $36 \%$ de trabajadores de la industria y para el año 2017 siete empresas concentran el 39\%. En contraste con los resultados encontrados, Blomström (1986) realizó un estudio que toca de manera tangencial la discusión sobre derramas pero que se refiere más a la forma en que el IED influía sobre la concentración del mercado en el sector manufacturero mexicano. La participación extranjera no fue significativa respecto a la concentración en términos estadísticos cuando se encontraba asociada con la variable de escala. Los resultados encontrados sugerían que la IED de las firmas multinacionales influía positivamente sobre la concentración en México independientemente de otras variables, pero tales hallazgos no permitieron hacer conclusiones definitivas. Por un lado, se sostiene que las empresas extranjeras imponen una mayor competencia a las empresas locales aumentando las barreras de entrada y por otro, las empresas multinacionales aumentan la concentración al introducir tecnologías de gran escala e intensidad de capital elevada. La ambigüedad de los resultados hacía posible argumentar que las empresas extranjeras aumentaban la concentración en los mercados manufactureros al introducir tecnologías de gran escala y al utilizar una intensidad elevada del capital. Pero también era posible el argumentar lo contrario, es decir, que las empresas extranjeras no causaban la alta concentración, sino que, precisamente, era la elevada concentración la que atraía a filiales de multinacionales. En resumen, la relación entre la IED y la concentra- 
ción del mercado intentaba dar un mejor soporte empírico que permitiera explicar la ocurrencia o no de las derramas de eficiencia con base en una relación estadísticamente significativa clara ya sea en sentido positivo o negativo entre ambas variables.

Estudios de spillovers de productividad generalmente utilizan como base de datos promedios por ramas industriales. A diferencia del presente estudio que genera los datos a nivel particular de cada empresa y cuya variación es propia de su rendimiento y rol en el mercado, en lugar de aspectos agregados cuando se trabaja por ramas o sectores industriales.

Reyes, Romoy Bazdresch (2004) señalan que para algunos autores el resultado de este tipo de medir la eficiencia técnica mediante la productividad laboral es puesto en cuestión debido a que la mejor productividad de las plantas domésticas podía deberse en parte a otros aspectos entre los que figura, por ejemplo, los ciclos económicos de las diversas actividades industriales, variaciones en cuanto a la utilización de los factores productivos, diferencias en el grado de utilización de la capacidad instalada, políticas públicas proteccionistas, subsidios e incluso la propia inercia de la productividad laboral, además de otro factores inobservables, tan sólo por citar algunos.

Del mismo modo, consideran presencia de endogeneidad en el sentido que, estimar una regresión para determinar la ocurrencia de derramas de productividad de las empresas extranjeras hacia las domésticas en función de un conjunto de variables independientes, no asegura con certeza que un incremento de la productividad de las empresas domésticas se deba enteramente a la presencia extranjera. Bien puede ser el caso, que las empresas extranjeras se hayan establecido en sectores con mayores niveles de productividad.

En ese sentido es importante aclarar que la evidencia mostrada en el presente estudio, así como por los autores descritos anteriormente sólo han podido decir si la evidencia encontrada está en línea o no con la noción de derramas de acuerdo a mediciones de causalidad descrita en la teoría.

Los resultados para encontrar existencia de spillovers de productividad se muestra en la tabla 9. La evidencia de spillovers es observar si las 
regresoras definidas ejercen influencia positiva en la variable explicativa definida como productividad solamente para las empresas nacionales.

Las variables ventas extranjeras (VTASEXT)para ambos periodos tienen signo positivo, pero no significativo. La variable que indica presencia de trabajadores calificados (TRAEXT)muestra signo negativo y no significativo. Las variables (ROBVEXT)que verifican la robustez de la presencia de spillovers para ambos años tienen impacto positivo en la productividad de las empresas nacionales y son estadísticamente significativo. Los resultados demuestran que las empresas con acciones extranjeras mayores o iguales a $50 \%$ presentan spillovers de productividad hacia las empresas nacionales.

Tabla 9

Estimación de spillovers de productividad

\begin{tabular}{|lrr|}
\hline & 2016 & 2017 \\
\hline VTASEXT & 8.712 & 12.262 \\
& $\mathrm{e}=12.474$ & $\mathrm{e}=11.673$ \\
& $(0.491)^{*}$ & $(0.302)^{*}$ \\
\hline TRAEXT & -8.801 & -11.887 \\
& $\mathrm{e}=11.783$ & $\mathrm{e}=8.929$ \\
& $(0.461)^{*}$ & $(0.193)^{*}$ \\
\hline ROBVEXT & 18.622 & 25.293 \\
& $\mathrm{ee}=6.222$ & $\mathrm{ee}=8.172$ \\
& $(0.006)^{*}$ & $(0.004)^{*}$ \\
\hline ROBTEXT & -27.777 & -17.860 \\
& $\mathrm{ee}=10.814$ & $\mathrm{ee}=7.775$ \\
& $(0.016)^{*}$ & $(0.029)^{*}$ \\
\hline Constante & 5.413 & 5.53 \\
& $\mathrm{ee}=0.333$ & $\mathrm{ee}=0.344$ \\
Pruebas de diagnóstico & $(0.000)^{*}$ & $(0.000)^{*}$ \\
$\mathrm{~J} / \mathrm{B}$ & & \\
White & {$[0.98]$} & {$[1.56]$} \\
Ramsey & {$[0.93]$} & {$[0.78]$} \\
$\mathrm{R}^{2}$ & {$[0.67]$} & {$[0.84]$} \\
F & 0.3225 & 0.3491 \\
\hline
\end{tabular}

$\mathrm{R}^{2}=$ Bondad de ajuste del modelo

$\mathrm{F}=$ Prueba de significancia de las variables en conjunto

* = Significancia al 95\% de confianza

$\mathrm{J} / \mathrm{B}=$ Prueba de Jarque Bera de Normalidad

White = Prueba de white de heteroscedasticidad

Ramsey = Prueba de forma funcional

Fuente. Elaboración propia

En relación a la variable (ROBTEXT) para ambos años tienen impacto negativo en la productividad de las empresas y son estadísticamente significativos. El resultado muestra que un incremento en la mano de obra 
calificada en las empresas de accionariado extranjera reduce la productividad de las empresas nacionales.

En relación a las pruebas de diagnósticos para verificar la no violación a los supuestos del MCRL, la prueba Jarque Bera y el test de White demuestran que los residuos presentan distribución normal y presencia de homoscedasticidad, por lo que los coeficientes calculados son estimadores insesgados y eficientes. La prueba funcional Reset-Ramsey reporta valores menores a la tabla $F$ en los respectivos grados de libertad, por lo que se acepta la hipótesis nula de no variables omitida en el modelo. Finalmente, el valor F permite descartar la hipótesis nula que todos los coeficientes de las variables explicativas son cero.

Los resultados parciales encontrados son similares a lo encontrado por Grether, JM (1999) quien encontró que en México el capital extranjero tiene una influencia positiva en la eficiencia productiva a nivel de planta, pero no produce efectos de contagio significativos a nivel sectorial. En contraste, Kokko, Tansini, yZeján (1994) en estudios sobre spillovers de IED en la industria manufacturera uruguaya indican que el resultado más notable es el signo positivo, y significativo del parámetro correspondiente a la participación de las empresas con participación de capital extranjero (PEXVT), indicando que su participación tiene un efecto positivo sobre la productividad de las empresas nacionales.

Resultados encontrados por Aitken y Harrison (1999) en 3,955 plantas manufactureras en Venezuela de 1976 a 1980. Primero, los investigadores encontraron que los aumentos en la participación de capital extranjero están correlacionados con los aumentos en la productividad para las plantas receptoras con menos de 50 empleados, lo que sugiere que estas plantas se benefician de las ventajas productivas de propietarios extranjeros. En segundo lugar, encontraron que los aumentos en la propiedad extranjera afectan negativamente la productividad de empresas de propiedad totalmente nacional en la misma industria. Estos efectos negativos son grandes y robustos para las especificaciones del modelo alternativo. Aunque estudios previos generalmente encontraron efectos positivos, demostraron que estos resultados pueden explicarse por la tendencia de las multinacionales a ubicarse en sectores más productivos e invertir en plantas más productivas. Concluyen que hay beneficios de la inversión ex- 
tranjera, pero que tales beneficios parecen estar internalizados por empresas conjuntas. Finalmente, no encontraron evidencia que respalde la existencia de "derrames" de tecnología de firmas extranjeras a firmas de propiedad nacional.

\section{Conclusiones}

Los hallazgos encontrados muestran que la productividad laboral de las empresas con capital extranjero presenta mayores niveles de productividad que las empresas nacionales.

A fin de determinar la existencia de spillovers desde las empresas extranjeras hacia las empresas locales los resultados no son contundentes. Se verifica que existe spillovers de productividad desde las empresas con capital extranjero hacia las empresas nacionales en aquellas empresas que tienen al menos 50\% de capital extranjero. Sin embargo, las empresas con $15 \%$ de capital extranjero muestra impacto positivo, pero estadísticamente no son significativo. Una posible interpretación es que las empresas con capital extranjero mayor al 50\% se encuentran en sectores rentables.

La presencia de personal calificado impacta positivamente en la productividad laboral. Esta presencia fue medida como el cociente entre trabajadores de cuello blanco (empleados, funcionarios y ejecutivos) y trabajadores de cuello azul (obreros).

La concentración de mercado representado por el porcentaje de trabajadores de cada empresa frente al total de la industria tiene un efecto negativo en la productividad. En efecto, un análisis sencillo permite verificar que para el año 2016 seis empresas concentran el 36\% de trabajadores de la industria y para el año 2017 siete empresas concentran el 39\%.

Las pruebas de diagnóstico para comprobar que el modelo cumpla con los supuestos de correcta especificación reflejan que el modelo empírico estimado no presenta problemas de especificación.

El presente estudio es relevante para el análisis de las derramas porque permitió comparar el desempeño de las empresas domésticas y las extranjeras establecidas en el Perú. Tales diferencias invitaban a probar 
cuál era el impacto de las empresas de capital extranjero sobre las de propiedad nacional, en particular, si se confirmaba la posibilidad de ocurrencia de derramas resultantes de la ÍED.

\section{Recomendaciones}

El presente estudio respondió a la interrogante que buscaban encontrar evidencia sobre el rol de las inversiones extranjeras directas mediante mecanismos de spillovers en la productividad en el Perú. Mediante un análisis de corte transversal para ambos periodos y el uso de un modelo de regresión lineal múltiple con presencia de variables dicotómicas para caracterizar las variables de estudio, los resultados sobre la relación positiva y estadísticamente significativa entre la productividad del trabajo de las empresas nacionales y la presencia de capitales extranjeros permiten concluir de manera parcial la existencia de desbordamientos de tecnologías, habilidades, procesos, mejoras organizacionales, entre otras.

Sin embargo, es importante buscar cuales son los mecanismos a través de los cuales se producen estas transmisiones. Un análisis sugerido para estudios posteriores es medir la productividad a nivel interindustrial que establecen las empresas multinacionales con proveedores y clientes locales (encadenamientos hacia adelante y hacia atrás), ya que estas relaciones podrían ser importantes canales de difusión de externalidades de IED.

Por otro lado, se recomienda extender el estudio para periodos más extensos de manera que permita adoptar técnicas econométricas combinadas en contraste con los resultados encontrados.

Debido a la menor productividad de las empresas nacionales frente a las empresas con presencia de capitales extranjeros es necesario fortalecer con políticas sectoriales los niveles de productividad y competitividad de las empresas de bandera nacional. La balanza de pagos muestra que las salidas de divisas por repatriación de utilidades de las empresas con capital extranjero superan los flujos de IED hacia el Perú. Existen dos explicaciones tentativas al respecto: Primero, las empresas extranjeras ya alcanzaron un nivel de madurez y, segundo, el carácter del capital es la de buscar en el mercado mundial mejores alternativas de inversión. Esto 
conlleva a revisar los beneficios y/o exoneraciones que gozan las empresas de accionariado estatal.

Finalmente, una de las restricciones del estudio es la disponibilidad de base de datos relevantes al alcance de investigaciones futuras. Información sobre tecnologías aplicadas, horas de capacitaciones, salarios, etc., son algunos datos que permitirían medir correlaciones entre las distintas variables de estudio. En este aspecto, es necesaria la presencia del Estado en la búsqueda de mecanismos de difusión transparente y de fácil acceso a la comunidad investigadora.

\section{Referencias Bibliográficas}

Aitken, BJ, Harrison, A. yLipsey (1996). Wages and Foreign Ownership: A Comparative Study of Mexico, Venezuela, and the United States.Universidad de Pennsylvania ScholarlyCommons. Workingpaper No. 5102.

Aitken, BJ y Harrison, A.(1999). Do Domestic Firms Benefit from Direct Foreign Investment? Theamericaneconomicreview.

Álvarez, R (2002). IED en Chile y su impacto sobre la productividad. Universidad de Chile.

Atallah, S (2006). Reevaluando la transmisión de spillovers de la IED: un estudio de productividad para Colombia. Revista desarrollo y sociedad.

Blomström, M. (1989). Foreign Investment and Spillovers. Londres: Routledge.

Blomström, M y Koko, A. (2001). FDI, Human Capital and Esducation in Developing Countries Technical Metting.

Blomström, M y Persson, H (1983). Foreign Investment and spillover efficiency in an underdeveloped economy: Evidence from the Mexican manufacturing industry.

Blomstrom, M yWolff, E.(1994). Multinational corporations and productivity convergence in Mexico. Nberworkingpaper serie No. 3141.

Briseño, J y Neme, O. (2011). Repatriación de utilidades de la inversión extranjera directa: La relación entre México y Estados Unidos.

Dominguez, L y Brown, F. (2004). Evolución de la productividad en la industria mexicana: una aplicación con el método de Malmquist.

Fujii, D. (2004). Inversión extranjera y productividad en México. Red de Revista Científica de América Latina. 
Gorg, H y Greenaway, D. (2003). Much ado about nothing? Do domestic firms really benefit from foreign direct investment?

Grether, J (1999). Determinants of Technological Diffusion in Mexican Manufacturing: A Plant-Level Analysis.

Guerra-Borge, A (2001). Factores determinantes de la inversión extranjera: Introduccion a una teoría inexistente"

Jiménez, D y Rendón, H (2011). Inversión extranjera directa en la industria manufacturera colombiana y spillovers de productividad. Ensayos de economía No. 39.

Mamingi, N y Martin, K. (2018). La inversión extranjera directa y el crecimeinto en los países en desarrollo: El caso de los países de la organización de estados del caribe oriental. Revista de la Cepal N. 124.

Reyes, A. Romo, D y Bazdersch, C (2004). El estudio de las derramas de la inversión extranjera directa en la industria manufacturera mexicana. Una nota histórica. Documento de trabago 305 CIDE.

Ronderos, C. (2010). La inversión extranjera directa en Colombia y su relación con la competitividad. 2010. Bogota: Institituto universitario ESEADE.

Suanes, My Roca, O (2015). Inversión Extranjera Directa, Crecimiento Económico y Desigualdad en América Latina. Revista académica Scielo.

Villena, M. (2013). Estudio de medición del impacto de la inversión extranjera directa en la economía Chilena. Santiago de Chile: SCL Econometrics.

\section{(Footnotes)}

1 Los egresos privados corresponden a las utilidades de la inversión extranjera directa, los intereses de la deuda externa y los intereses por depósitos de no residentes en el país. 
